\title{
Aspect extraction on user textual reviews using multi-channel convolutional neural network
}

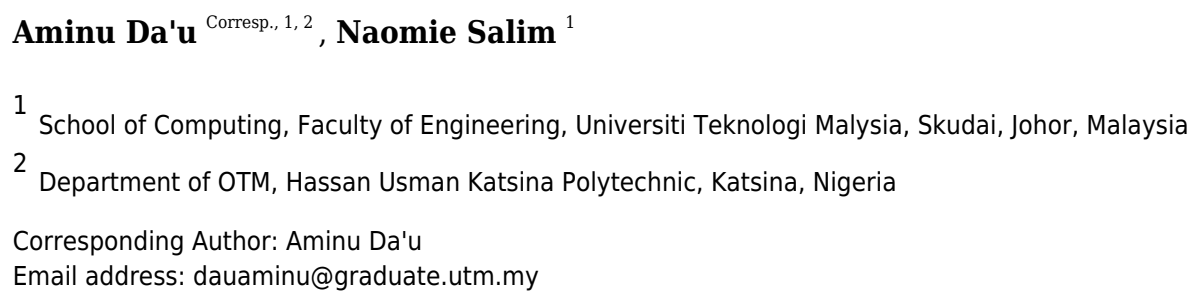

Aspect extraction is a subtask of sentiment analysis that deals with identifying opinion targets in an opinionated text. Existing approaches to aspect extraction typically rely on using handcrafted features, linear and integrated network architectures. Although these methods can achieve good performances, however, they are time-consuming and often very complicated. In real-life systems, a simple model with competitive results is generally more effective and preferable over complicated models. In this paper, we present a multichannel convolutional neural network for aspect extraction. The model consists of deep CNN (Convolutional Neural Network) with two input channels: Word embedding channel which aims to encode semantic information of the words and POS (part of speech) tag embedding channel, to facilitate the sequential tagging process. To get the vector representation of words, we initialized the word embedding channel and POS channel using pre-trained word2vec and one-hot-vector of POS tags respectively. Both the word embedding and POS embedding vectors were fed into the convolutional layer and concatenated to a one-dimensional vector which is finally pooled and processed using a softmax function for sequence labeling. We finally conducted a series of experiments using four different datasets. The results indicated better performance compared to the baseline models. 
1 Aspect extraction on user textual reviews using multi-

2 channel convolutional neural network

3

\author{
Aminu Da'u ${ }^{a b}$, Naomie Salim ${ }^{a}$ \\ ${ }^{a}$ School of Computing, Faculty of Engineering Universiti Technologi Malaysia, \\ Malaysia. \\ ${ }^{b}$ Department of OTM, Hassan Usman Katsina Polytechinc, Katsina, Nigeria. \\ *Corresponding Author Tel: $\mathbf{+ 6 0 1 8 7 9 2 2 5 2 8}$ \\ Email Addresses: dauaminu@gmail.com, dauaminu@graduate.utm.my (A.Da'u), \\ Naomie@utm.my ( N. Salim)
}

\begin{abstract}
Aspect extraction is a subtask of sentiment analysis that deals with identifying opinion targets in an opinionated text. Existing approaches to aspect extraction typically rely on using handcrafted features, linear and integrated network architectures. Although these methods can achieve good performances, however, they are time-consuming and often very complicated. In real-life systems, a simple model with competitive results is generally more effective and preferable over complicated models. In this paper, we present a multichannel convolutional neural network for aspect extraction. The model consists of deep CNN (Convolutional Neural Network) with two input channels: Word embedding channel which aims to encode semantic information of the words and POS (part of speech) tag embedding channel, to facilitate the sequential tagging process. To get the vector representation of words, we initialized the word embedding channel and POS channel using pre-trained word2vec and one-hot-vector of POS tags respectively. Both the word embedding and POS embedding vectors were fed into the convolutional layer and concatenated to a one-dimensional vector which is finally pooled and processed using a softmax function for sequence labeling. We finally conducted a series of experiments using four different datasets. The results indicated better performance compared to the baseline models.
\end{abstract}




\section{Introduction}

With the growth of textual information on the web, aspect-based sentiment analysis has been widely studied, thereby attracting many attentions in the research community. One of the important subtasks of aspect-based sentiment analysis is aspect extraction which is simply the act of extracting attributes of an entity about which opinions are expressed[1]. Aspect extraction can generally be performed using either unsupervised [2], [3] or supervised methods [4]-[6]. For many years, the state-of-the-art methods of aspect extraction basically depend on the CRF (conditional random fields) [4], RNN (recurrent neural network) [7], linguistic patterns and syntactic rules [2], [8]. Both of these approaches have their own shortcomings. For example, CRF is typically linear in nature. Thus, it requires a large number of datasets to effectively work. RNNs are generally not effective in predicting word labels or phrases that are determined by the context due to their feedback nature. Syntactic rules and linguistic patterns need to be handcrafted and their accuracy generally depends on the grammatical accuracy of the sentences.

To address the aforementioned issues among others, few approaches have been proposed to exploit deep CNN architectures to improve the performance of the aspect extraction models [5], [9]. These models do not usually require predefined features to be manually handpicked, instead, they can automatically learn sophisticated features from their datasets. Generally, words are usually represented in the form of a vector and the extraction of the feature is left to the network. Consequently, words with similar semantics can be mapped using these models to nearby locations in their coordinate systems.

Even though these approaches have shown better performances than their prior approaches, however, there are some important issues worth to be considered for further improvement: First, most of the existing approaches typically used only general pre-trained word embeddings such as Google Word2vec or Glove embeddings as the main semantic feature for the aspect extraction. Although word embeddings have shown effectiveness in capturing both syntactic and semantic information of words. However, in some cases, due to the distributional hypothesis, word embeddings alone fail to efficiently capture the syntactic information of some aspect terms, for example, in the latent space, bad and good are typically mapped together as neighbors while analyzing these words is very critical in aspect classification. Moreover, due to the complexity of aspect extraction task, fine-grained embeddings are particularly important to achieve a better performance [10]. Therefore, we urge that using a domain-specific embedding is very crucial for information extraction performance. Thus, in this paper, we exploit both the general and domain-specific embeddings to examine which embeddings are superior over the other.

Additionally, most of the previous CNN based aspect extraction models are either stacked [11] or integrated with other models such as LSTM ( Long Short Term Memory) [12]. These consequently increase the complexity of the model parameters. Although these may improve the 
model performance, however, according to Blumer et al. [13], in real-world applications, a simple model is always preferred and more useful over the complicated model. This is particularly important when a model is used for a real-life situation such as chatbot in which a complex model will retard the inferential performance of the model. Thus, achieving a competitive performance while ensuring a simple architecture without manually crafting features and much complexity is always a crucial direction to explore. This paper proposes to achieve such a goal.

Motivated by the above-mentioned issues, this paper proposes an aspect extraction model based on an MCNN (Multichannel Convolutional Neural Network) leveraging two different embedding layers: word embedding and POS tag embedding layer. To achieve a simple architecture while ensuring a competitive performance, we propose a purely CNN model for sequential labeling. CNN model which is nonlinear network architecture can fit data more easily with relatively few parameters. The major contributions of the proposed model can be summarized as follows:

1. We introduced an MCNN model for aspect extraction leveraging two different input channels: word embeddings and POS Tag embeddings channel to encode the contextual information and enhance sequential tagging of words respectively.

2. We investigated the importance of using domain-specific embeddings over the generalpurpose word embeddings in aspect extraction.

3. We conducted a series of experiments on the SemEval challenge datasets [29] [30] [31] and showed that our approach outperformed the baseline methods with significant gains across all the datasets.

The remainder of the paper is arranged as follows. Section 2, related work, section 3 the proposed model, section 4 experimental study, section 5 results and discussion and section 6 conclusion and future direction.

\section{Related Work}

Aspect extraction as the subtask of aspect-based sentiment analysis has been widely studied by many researchers. One of the earliest studies was conducted by Hu and Liu [14] to propose a rule-based method for the explicit aspect categorization. This method was later improved by many approaches among which include the work of Popescu and Etzioni [8] who used point-wise mutual information between the product class and noun phrase for product feature extraction.

Generally, aspect extraction can be performed using either unsupervised [2], [3], [8], [15] or supervised method [4]-[6]. Our proposed work particularly focuses on the supervised methods which treat aspect extraction task as a sequence labeling problem. The traditional supervised methods are mainly based on HMM (Hidden Marcok Model) [16] and CRF [4]. With the recent success of deep learning in different areas such as image classification and pattern 
112 recognition, several approaches have been proposed to exploit deep learning methods for the

113 aspect extraction. For instance, Wang et al. [17] employed RMB (Restricted Boltzmann Machine)

114 model to jointly address the problem of sentiment-aspect extraction. Irsoy and Cardie [7] utilized

115 RNN and demonstrated its superior performance over the CRF based models for aspect

116 extraction. This method was later improved by Pengfei et al. [18]. They applied more

117 sophisticated variants of the RNN using fine-tuned word vectors and additional linguistic features

118 for better improvement. To tag each word with non-aspect or aspect label, a multi-layer

119 convolutional neural network was proposed by Poria et al. [5]. The authors used syntactic and

120 linguistic patterns to improve the accuracy of the model.

121 For further improvement, the attention-based model has been used for aspect extraction

122 to learn the representation of the informative words in text review [19], [20] [30,31]. Tree-based

123 methods have been shown effective for improving the performance of the aspect extraction

124 model. For instance, Yin et al. [10] introduced a dependency path approach in which both the

125 dependency and linear contextual information are considered for the word representation. A

126 similar method was proposed by Wang et al. [21] to exploit dependency tree and CRF for better

127 co-extraction of aspect and opinion terms. Li et al. [22], [23] also exploited deep learning for co-

128 extraction of the aspect and opinion terms. Recently, a tree-based convolutional neural network

129 was introduced by Ye et al. [11]. They applied tree-based convolution over a sentence

130 dependency parse tree. Lou et al. [24] proposed an end-to-end method to integrate BiLSTM, CRF

131 and word embeddings for aspect term extraction.

Our approach is closely related to the work of Xu et al. [25] in which a double embeddings method has been used to model aspect extraction using two different in-domain word embeddings. However, this method has a drawback in that it solely relies on the word embedding as the main feature and ignore to utilize the POS tag for the sequential tagging. In our approach, POS tags features are utilized in addition to the word embedding features to improve the model performance. Furthermore, unlike the previous methods, we particularly use two different channels as the input to the convolutional network architecture. We used both general and domain-specific embedding in the first channel specifically to capture the syntactic and semantic information of the word, and POS tag embedding in the second channel to specifically improve the sequential labeling of the aspects. To the best of our knowledge, this is the first work to use a multi-channel CNN architecture leveraging both word embeddings and POS tag embeddings in different channels for better performance of the aspect extraction model.

\section{Our Model}

Figure 1 illustrates the proposed MCNN architecture. The model is based on the CNN structure proposed in [26]. Specifically, the proposed model is made up of two input layers: word 
150

151

152

153

154

155

156

157

158

159

160

161

162

163

164

165

166

167

168

169

170

171

172

173

174

175

176

177

178

179

180

181

182

183

184

185

186 pooling layer, RLU (rectified linear unit) optimizer, a fully connected layer, and a softmax classifier to predict the multi-class labels of the aspects with labelling space, $Y=\{B, I, O\}$ with "I", " $O$ " and " $\mathrm{B}$ " representing Inside, Outside or Beginning of the aspect term respectively. Detail of the model is described in the following subsections:

(Figure1: Overview of the MCNN Architecture)

\subsection{Input Channels}

The model typically comprises two sets of vectors, each of which is an input channel to the network [26]. For the word embedding channel, the main idea is to capture the semantic information of the words. For that, we use both general and domain-specific embeddings. For the general embeddings, we particularly use a pre-trained embedding trained on 100 billion words of google corpus [27]. While for the domain-specific embeddings, we specifically train CBOW (continues bag of the word) [27] model on the Amazon and Yelp reviews for the laptop and restaurant domain respectively. In this case, each word was encoded as 300-dimensional vectors. We use word padding to make sure that all sentences are of the same length. To capture the contextual features of the words, $\mathrm{i}$-th words are mapped to a k-dimensional embedding. The semantic feature of a sentence of length $\mathrm{n}$ is given as $|X|_{1}^{n}=\left\{x_{1} \ldots \ldots, x_{n}\right\}, X \in R^{K}$.

For the POS Tag embeddings, the main idea is to improve the aspect extraction process based on the POS tagging. Specifically, we employ one hot vector in which each tag is transformed into a K dimensional vector. Similar to [28], we use a Stanford POS Tagger with 45 tags. These are encoded as 45-dimensional vector and represented as a matrix. This can be represented as: | $\left.S\right|_{1} ^{n}=\left\{s_{1} \ldots \ldots, s_{n}\right\}, \in R^{45}$.

\subsection{Convolutional Layer}

After all the textual information is encoded into vectors and zero padding is applied to make all the embedding channels of the same length, the convolution operations are then applied to generate local features. Thus, the main purpose of the convolutional layer is to extract local features from the embedding layer. Here we use two different filter sizes for POS feature $\boldsymbol{P}$ and Semantic Feature $\boldsymbol{Z}$ accordingly. Typically, convolution is a dot product involving filters with weights $W \in R^{h k}$ and a vector of h-gram in a sentence [26]. Let $w_{p} \in R^{h k}$ and $w_{z} \in R^{h K}$ be filter applied to $h$-gram for the matrix $\boldsymbol{P}$ and matrix $\mathbf{Z}$ respectively. Then the features generated can be given as:

$C_{i}=f\left(w \cdot x_{i+h}+b\right)$ 
187 Where $\mathrm{f}$ is a nonlinear function (such as hyperbolic tangent or ReLU), $b$ stands for a bias term.

188 This is applied to each window, $\left[x_{1: h}, x_{2: h+1}, \cdots x_{n-h: n,}\right]$. With the $w_{p} \in R^{n-k+1}$ and $w_{z} \in$ $189 R^{n-k+1}$, for the matrix $P$ and matrix $Z$ respectively. The features generated for $p$ is given by:

$190 c_{p}=\left[c_{1}^{p}, c_{2}^{p} \cdots c_{n-h+1}^{p}\right]$

191 And similarly, the feature map for matrix $Z$, is given as

$192 c_{z}=\left[c_{1}^{Z}, c_{2}^{Z} \ldots . c_{n-h+1}^{Z}\right]$

193 However, it is worth to mention that, different semantic and POS features can be extracted using 194 several filters.

195

196

197

198

199

200

201

202

203

204

205

206

207

208

209

210

211

212

213

214

215

216

217

218

219

220

221

222

223

\subsection{Max Pooling Layer}

Pooling operation is basically aimed to reduce the feature resolution maps by applying a pooling function to several units in a local region of a size based on a parameter known as pooling size. The pooling operation generally serves as generalizations over the features captured from the convolutional operation. Thus, the basic idea behind utilizing max poling layer is to extract the most salient features from the convolutional layer. Typically, pooling layer takes the maximum element in each generated feature map. This can be given as:

$$
\check{\mathrm{C}}_{p}=\max \left[c_{1}^{p}, c_{2}^{p} \ldots c^{p}{ }_{n-h+1}\right] \text { and } \check{\mathrm{C}}_{z}=\max \left[c_{1}^{Z}, c_{2}^{Z} \ldots c^{p}{ }_{n-h+1}\right] \text { for } \boldsymbol{P} \text { and } \boldsymbol{Z} \text { respectively. }
$$

When the max pooling is applied, the final maximum feature is generated by concatenating the semantic and POS features using a filter. This can be given as $C=\check{C}_{p} \oplus \check{C}_{z}$. Where $\oplus$ is the concatenation operator. As we use several features for the POS and semantic features, we have the final feature as:

$C=\check{C}_{p}^{1} \oplus \ldots . . \oplus \check{C}_{p}^{n} \oplus \check{C}_{z}^{m} \oplus \ldots . . \oplus \check{C}_{z}^{m}$

Where $\boldsymbol{n}$ and $\boldsymbol{m}$ are the filters for semantic and POS features respectively.

\subsection{Output layer}

Here we finally apply the softmax classifier to generate the probability distribution over given aspects. The main idea of the softmax function is to carry out a classification process over the high-level features generated from the convolution operation and pooling layers. In this case, the softmax function is used to find the probability distribution for all the output labels. Here, we specifically treat the aspect extraction as a sequence labeling process. Particularly we apply IOB scheme to indicate our aspect annotations as a tag sequence. Each word in the text is assigned with one of the 3 tags: I, O or B indicating beginning, Inside or Outside of an aspect term respectively. 


\section{Model Variations}

In order to evaluate our model, we conduct a series of experiments with different settings of the model.

- MCNN-Random: To assess the impacts of word embeddings, the word embedding channel is randomly initialized while the input channel containing the POS Tag embeddings is ignored, meaning that only the randomized word embeddings channel is considered for training.

- MCNN+W2V: Here, the word embedding layer is initialized with a pre-trained word2vec and optimized during training. Particularly, we used a general purpose word embeddings trained on the Google corpus [27].

- $M C N N+W 2 V 2:$ This is similar to the MCNN+W2V setting, however, instead of using the general pre-trained word embedding, we use a domain-specific word2vec trained on either Amazon or Yelp review datasets. This is specifically aimed to assess the impacts of the domain-specific word embeddings compared to the general word embeddings.

- MCNN+W2V+POS: In this case, all the two input channels are considered for the training and optimization process. Specifically, we use the general word embeddings in one channel and POS Tag embeddings in the other channel. However, the model parameters are fine-tuned during optimization

- MCNN+W2V2+POS: This is similar to MCNN-W2V+POS variant, however, in this case, instead of applying a general pre-trained word2vec, a domain specific word embedding is used. All the parameters are fine-tuned.

\section{Experimental Study}

In this section, we first present a description of the datasets used, then provide a detailed experimental procedure for evaluating the performance of the proposed approach and finally make a comparison against the baseline methods. We use Recall, Precision and F1 score as the evaluation metrics to evaluate the performance of the model. These metrics have been previously used in several relevant works [5], [8].

\subsection{Dataset}

To evaluate the performance of the model, we utilized four different benchmark datasets. The datasets which comprise training and test snippets were collected manually and made available by the organizers for the SemEval competitions. The first two datasets are from SemEval2014 [29] comprising reviews from laptop and restaurant domains respectively, while the third and fourth datasets are from semeval2015 [30] and SemEval2016 [31] respectively containing reviews from restaurant domain. These datasets comprise review sentences with 
263

264

265

266

267

268

269

270

271

272

273

274

275

276

277

278

279

280

281

282

283

284

285

286

287

288

289

290

291

292

293

294

295

296

297

298

299

300

aspect terms labelled as spans of characters. Table 1 and Table 2 show the statistics of the datasets and a typical example of the aspect terms distribution of the laptop and restaurant domains respectively.

(Table 1: SemEval challenge datasets showing the number of sentences and the aspect terms. L and $\mathrm{R}$ represent laptop and restaurant domains respectively)

(Figure 2: Example of Aspect and Aspect Terms word distribution in the laptop and restaurant domains.)

In order to initialize the word vectors, we particularly exploit two different word embeddings: (1) General embeddings in which we use pre-trained Google word2vec trained on 100 billion words of google news corpus [27] using CBOW (continues-bag-of-word) architecture. (2) Domain-specific embeddings trained on the restaurant review from the Yelp datasets, and electronics reviews from the Amazon datasets, for the restaurant and the laptop domains respectively. The ${ }^{1}$ Yelp and Amazon[32] review datasets contain 2.2 million and 142.8 million reviews respectively. We use Gensim which has the implementation of CBOW to train all the datasets. Words that appear less than 5 times in the review are replaced with <other> token.

\subsection{Preprocessing}

We carry out preprocessing with the aim of obtaining a clean and structured textual review. Specifically, we convert all the reviews into lower case comprised of only English texts and split the text into separate sentences. We apply noise removal strategy which includes removal of words with special characters, stop words, alphanumeric characters and words that have a length less than or equal to 1.

${ }^{1}$ https://www.yelp.com/dataset/

\subsection{Experimental Setup}

We use 5-folds cross-validation strategy to choose the hyperparameters. Specifically, we choose three filter size of $(3,4,5)$, with 100 feature maps. We used a max pooling layer after each convolutional layer. As we wanted to tag each word, we use 1 as the stride for each convolutional layer. To tackle the issue of the parameter overfitting, we utilized drop out regularization on the penultimate layer with $L 2$ constraints of 3 . The training is conducted using 
301

302

303

304

305

306

307

308

309

310

311

312

313

314

315

316

317

318

319

320

321

322

323

324

325

326

327

328

329

330

331

332

333

334

335

336

stochastic gradient descendent over shuffled mini batches of size 64 and a dropout rate of 0.5. We apply ReLU for all the datasets and used 128 to be the size of the hidden rate. These values were chosen based on the careful grid search on the validation subset.

To better assess the performance of the proposed model, we first identify the best performing settings of the model (as described in section $3 \mathrm{E}$ ) and then make a further comparison with the following baselines models:

- DLIREC [33]: The winning system in the SemEval2014 competition (subtask 1) which employ a variety of lexical and semantic features derived from NLP source to improve the performance of the model

- IHSR \& D [34]: Another top winning system in the semeval2014 competition which typically exploits CRF and additional features including lexical and statistical features for improving the performance.

- NLANGP [35]: The top system for semeval2016 challenge for the restaurant domain.

- WDEmb [10]: A dependency-based approach integrated CRF with path embedding for aspect term extraction.

- BiLSTM-CNN-CRF [36]: This is an integrated deep learning based model with the CRF layer. It is the state-of-the-art aspect extraction approach from the Named Entity Recognition Community.

- RNCRF [21]: This model jointly uses CRF and a dependency-based recursive neural network for co-extracting aspects and opinion terms. The method also exploits additional handcrafted features.

- CMLA [20]: A multi-layer coupled-attention model for opinion and aspect terms coextraction.

- MIN [23]: A multitask learning approach that exploits lexicons and dependency rules to jointly perform co-extraction of aspect terms and opinion terms. It uses two different LSTMs for the polarity classification of sentences.

- DTBCSNN [11]: A dependency tree based convolutional stacked neural network which used the inference layer for aspect extraction.

- DE-CNN [25]: A CNN based model exploiting double embeddings for aspect extraction.

- BiDTreeCRF [24]: A tree-based deep learning approach which uses bidirectional LSTM and the CRF layer for improving aspect extraction.

(Table 3: Comparison results of our model in terms of F1 scores (\%) with state-of-the-art methods) 
337 (Table 4: Comparison results among the different variations of our model in terms of recall, 338 precision, and F1 score performance).

\section{Results and Discussion}

342

Table 3 shows the results of the proposed model compared to the baseline models. Here, the results of the best two settings of the model were recorded for each dataset. It can be shown that the best performing variants of the proposed model significantly outperform the state of art approaches. The statistical t-test shows the improvement is significant at the confidence level of $95 \%$.

Compared to the best-performing systems in the SemEval competitions, our model performs better than HIS_RD and DLIREC with gains of $6.08 \%, 7.27 \%$ and $6.85 \%, 2.88 \%$ F1 score on the semEval2014-L and SemEval2014-R datasets respectively. Similarly, our approach also achieves significant gains against NLANGP by $5.72 \%$ and $3.37 \%$ F1 score on the SemEval2005-R and SemEval2016-R respectively. It can be observed that even the WDemb approach which exploits word dependency with additional embedding, still our model achieved significant gains compared to the model across all the datasets. One can also notice from table 3 that, our model outperforms MIN which is a multitasking approach, with a gain of 3.05\%, 1.6\%, $2.11 \%$, and $2.27 \%$ F1 score on the SemEval2014-L, SemEval2014-R, SemEval2015-R and SemEval2016-R datasets respectively. Our model also outperforms CMLA which is a multilayer approach by $2.83 \%$ F1 score on the semeval2014-L datasets.

Despite exploiting the additional handcrafted features by RNCR+F and DTBCSNN, still, our approach achieves $2.21 \%, 1.96 \%$ and 4,97\%, 2.92\% F1 score gains over the two approaches on the semeval2014-L and semeval-2014-R datasets respectively. Moreover, our model outperforms the recent tree-base bidirectional method, BidTreeCRF by $0.06 \%, 2.06 \%, 2.01 \%$ and 1.22\% F1 score on the semeval2014-L, semeval2014-R, semeval2015-R and semeval2016-R datasets respectively. Compared to the double embedding CNN approach, DE-CCN which is the state-of-the-art double embedding approach, our model suffered a low performance on the semeval2014-L, however, it manages to achieve a gain of 1.34\% F1 score on the semeval2016-R datasets. This apparently shows the superior performance of our model over the DE-CNN model.

It can be observed from Table 4, that different settings of the model have different performances across the four different datasets. MCNN-WV2-POS performs better than all the other variants across all the datasets while the MCNN-random records relatively lowest performance except on the semeval2015-R where the MCNN-WV2-POS records the best results. This is likely due to the relatively smaller size of the semeval2015-R datasets. Similarly, one can notice from the Table 4, that in all the variants, the best results were recorded on the restaurant 
374

375

376

377

378

379

380

381

382

383

384

385

386

387

388

389

390

391

392

393

394

395

396

397

398

399

400

401

402

403

404

405

406

407

408

409

410

411

domain while relatively lower results are recorded on the laptop domain in all the datasets. This is likely due to the lower number of the aspects term contained in the restaurant domain than in the laptop review domain.

As can be seen from Table 4 and Figure 2, all the variants of our model with the exception of MCNN-random demonstrate relatively competitive results with significant improvement across all the domains. This specifically indicates the weakness of the randomly initialized word embeddings for the aspect extraction. This is because MCNN-random is randomly initialized while the other variants are particularly initialized with pre-trained word embeddings. This translates the importance of pre-trained word embeddings over the randomly initialized word embeddings. It is also shown from the results that using domain-specific word embedding for both laptop and restaurant domains performs better than the general word embeddings (Google embeddings) initialization. This supports the intuition that domain-specific embeddings typically contain opinion specific information related to a particular domain (Laptop or restaurant) which helps to perform better than the general word embeddings which are merely trained on the google news corpus which is typically composed of textual reviews about the commonly discussed matters on the news.

One can observe from figure 3 that in both laptop and restaurant domain the model suffers from low recall, meaning that it missed some vital aspect terms. However, using the POS tag which is an important linguistic feature help to overcome some drawbacks thereby improving the performance of the model. This specifically indicates the importance of using POS tags features in addition to pre-trained word embeddings in aspect term extraction.

We further conduct an experiment to assess the sensitivity of the model towards word embeddings dimensions. We specifically use different word embedding dimensions from 50 to 375 with the intervals of 25 . i.e. $\{50,75,100,125,150,175,200,225,250,275,300,325,350$, $375\}$. The laptop domain uses embeddings trained on the Amazon reviews and restaurant domain use the embeddings trained on the Yelp reviews datasets. Figure 4 shows the experimental results on the MCNN-WV2 variant. The results indicate the highest performance at around 300 dimensions and relatively remains stable above 150 . This particularly implies the insensitivity of the model towards the dimension of word embeddings provided it is within the appropriate range such as 100 to 375 .

It is clear that two key factors are basically the reasons behind the good performance of our model compared to the baseline methods: First, the POS tag embedding input layer which helps for better sequence labeling. The domain-specific pre-trained word embeddings which were trained on the target domain corpus of the review datasets. The advantage of our approach is that it is relatively uncomplicated and automatic that does not require any feature engineering. This saves time, cost and improves the high performance of the model.

(Figure 2: Performance results of the different model variants in terms of F1 score accuracy)

Peer] Comput. Sci. reviewing PDF | (CS-2018:11:33034:3:0:NEW 8 Apr 2019) 
412

413 (Figure 3: Performance of our model variants in terms of recall and precision (\%)

414

415

416

417

418

419

420

421

422

423

424

425

426

427

428

429

430

431

432

433

434

435

436

437

438

439

440

441

442

443

444

445

446

447

448

449

450

(Figure 4: F1 score (\%) of the MCNN-WV2-POS on different word embeddings dimension)

\section{Conclusion}

In this research, we proposed an aspect extraction approach using Deep MCNN leveraging two different channels namely, word embeddings and POS tag embeddings. We presented a series of experiments and the results on various datasets showed that our proposed approach outperformed the state-of-the-art methods. Our results support the previous findings that showed that pre-trained word vectors are always better than randomly initialized embeddings in deep learning based NLP tasks such as aspect extraction. It also reaffirms many of the previous findings which show that exploiting POS tag features improves the performance of NLP methods including aspect extraction. We also demonstrated the importance of using a domain specific word embedding over the general word embeddings. As a feature direction of the research, we think that, applying attention based deep learning model for improving aspect extraction is worth to explore and that integrating lexicon in the word embedding layer in the multichannel CNN is also another direction to explore.

\section{References}

[1] B. Liu, Sentiment Analysis and Opinion Mining, no. May. Morgan \& Claypool Publishers., 2012.

[2] Q. Guang and L. Bing, "Opinion Word Expansion and Target Extraction through Double Propagation," Comput. Linguist., no. July 2010, 2009.

[3] B. Wang and H. Wang, "Bootstrapping Both Product Features and Opinion Words from Chinese Customer Reviews with Cross-Inducing.," Ijcnlp, no. 60675035, pp. 289-295, 2008.

[4] J. Lafferty and A. Mccallum, "Conditional Random Fields: Probabilistic Models for Segmenting and Labeling Sequence Data," in Proceedings of the 18th International Conference on Machine Learning 2001 (ICML 2001), 2001, vol. 2001, no. June, pp. 282289.

[5] S. Poria, E. Cambria, and A. Gelbukh, "Aspect Extraction for Opinion Mining with a Deep Convolutional Neural Network," Knowledge-Based Syst., vol. 108, pp. 42-49, 2016.

[6] E. Cambria, "Affective Computing and Sentiment Analysis," IEEE Intell. Syst., vol. 31, no. 2, pp. 102-107, 2016.

[7] O. Irsoy and C. Cardie, "Opinion mining with deep recurrent neural networks," Proc. 2014 Conf. Empir. Methods Nat. Lang. Process., pp. 720-728, 2014.

[8] A. Popescu and O. Etzioni, "Extracting Product Features and Opinion from Reviews," Hum. Lang. Technol. Empir. Methods Nat. Lang. Process. Vancouver, Br. Columbia, pp. 339-346, 
451

452

453

454

455

456

457

458

459

460

461

462

463

464

465

466

467

468

469

470

471

472

473

474

475

476

477

478

479

480

481

482

483

484

485

486

487

488

489

490

491

492

493

494

2005.

[9] H. Xu, B. Liu, L. Shu, and P. S. Yu, "Double Embeddings and CNN-based Sequence Labeling for Aspect Extraction," in Proceedings ofthe 56th Annual Meeting ofthe Association for Computational Linguistics, 2018, pp. 592-598.

[10] Y. Yin, F. Wei, L. Dong, K. Xu, M. Zhang, and M. Zhou, "Unsupervised word and dependency path embeddings for aspect term extraction," in IJCAI International Joint Conference on Artificial Intelligence, 2016, vol. 2016-Janua, pp. 2979-2985.

[11] H. Ye, Z. Yan, and Z. Luo, "Dependency-Tree Based Convolutional Neural Networks for Aspect Term Extraction," no. April 2017, 2017.

[12] F. Dong, Y. Zhang, and J. Yang, "Attention-based Recurrent Convolutional Neural Network for Automatic Essay Scoring," Proc. 21st Conf. Comput. Nat. Lang. Learn. (CoNLL 2017), pp. 153-162, 2017.

[13] A. Blumer, A. Ehrenfeucht, D. Haussler, and M. K. Warmuth, "Occam's Razor," Inf. Process. Lett., vol. 24, no. 6, pp. 377-380, 1987.

[14] M. Hu and B. Liu, "Mining and summarizing customer reviews," Proc. 2004 ACM SIGKDD Int. Conf. Knowl. Discov. data Min. - KDD '04, p. 168, 2004.

[15] Z. Chen, A. Mukherjee, and B. Liu, "Aspect extraction with automated prior knowledge learning," 52nd Annu. Meet. Assoc. Comput. Linguist. ACL 2014 - Proc. Conf., vol. 1, pp. 347-358, 2014.

[16] W. Jin and H. H. Ho, "A novel lexicalized HMM-based learning framework for web opinion mining," in Proceedings of the 26th Annual International Conference on Machine Learning - ICML'09, 2009, pp. 1-8.

[17] L. Wang, K. Liu, Z. Cao, J. Zhao, and G. de Melo, "Sentiment-Aspect Extraction based on Restricted Boltzmann Machines," in Proceedings of the 53rd Annual Meeting of the Association for Computational Linguistics and the 7th International Joint Conference on Natural Language Processing (Volume 1: Long Papers), 2015, pp. 616-625.

[18] L. Pengfei, J. Shafiq, and M. Helen, "Fine- grained opinion mining with recurrent neural net- works and word embeddings," in . In Proceedings ofthe 2015 Conference on Empirical Methods in Natural Language Processing, 2015, pp. 1433-1443.

[19] P. Chen, Z. Sun, L. Bing, and W. Yang, "Recurrent Attention Network on Memory for Aspect Sentiment Analysis," in Proceedings of the 2017 Conference on Empirical Methods in Natural Language Processing, 2017, pp. 452-461.

[20] W. Wang, S. J. Pan, and D. Dahlmeier, "Multi-task memory networks for category-specific aspect and opinion terms co-extraction," Aaai, pp. 3316-3322, 2017.

[21] W. Wang, S. J. Pan, D. Dahlmeier, and X. Xiao, "Recursive Neural Conditional Random Fields for Aspect-based Sentiment Analysis," Proc. 2016 Conf. Empir. Methods Nat. Lang. Process., pp. 616-626, 2016.

[22] L. Xu, J. Lin, L. Wang, C. Yin, J. Wang, and J. W. Lamei Xu, Jin Lin, Lina Wang, Chinyong, "Deep Convolutional Neural Network based Approach for Aspect-based Sentiment Analysis," Adv. Sci. Technol. Lett., vol. 143, no. Ast, pp. 199-204, 2017.

[23] X. Li, W. Lam, E. Management, and H. Kong, "Deep Multi-Task Learning for Aspect Term Extraction," pp. 2886-2892, 2017.

[24] H. Luo, T. Li, B. Liu, B. Wang, and H. Unger, "Improving Aspect Term Extraction with Bidirectional Dependency Tree Representation," 2018. 
495

496

497

498

499

500

501

502

503

504

505

506

507

508

509

510

511

512

513

514

515

516

517

518

519

520

521

522

523

524

525

526

527

528

529
[25] H. Xu, B. Liu, L. Shu, and P. S. Yu, "Double Embeddings and CNN-based Sequence Labeling for Aspect Extraction," in Proceedings ofthe 56th Annual Meeting ofthe Association for Computational Linguistics, 2018, pp. 592-601.

[26] Y. Kim, "Convolutional Neural Networks for Sentence Classification," in proceedings of the 2014 Emperical Methods in Natural Language Processing (EMNLP), 2014, pp. 23-31.

[27] T. Mikolov, W. Yih, and G. Zweig, "Linguistic regularities in continuous space word representations," in Proceedings of NAACL-HLT, 2013, no. June, pp. 746-751.

[28] S. Jebbara and P. Cimiano, "Aspect-Based Relational Sentiment Analysis Using a Stacked Neural Network Architecture," in on Artificial Intelligence, 29 August-2 ..., 2016.

[29] M. Pontiki and J. Pavlopoulos, "SemEval-2014 Task 4: Aspect Based Sentiment Analysis," in Proceedings of the 8th International Workshop on Semantic Evaluation, 2014, no. SemEval, pp. 27-35.

[30] M. Pontiki, D. Galanis, and H. Papageorgiou, "SemEval-2015 Task 12 : Aspect Based Sentiment Analysis," 2015, p. Semeval.

[31] M. Pontiki, D. Galanis, H. Papageorgiou, I. Androutsopoulos, and Manandhar, "SemEval2016 Task 5 : Aspect Based Sentiment Analysis," in SemEval, 2016, pp. 19-30.

[32] J. McAuley and J. Leskovec, "Hidden factors and hidden topics: understanding rating dimensions with review text," Proc. 7th ACM Conf. Recomm. Syst., pp. 165-172, 2013.

[33] Z. Toh and W. Wang, "DLIREC: Aspect Term Extraction and Term Polarity Classification System," in Proceedings of the 8th International Workshop on Semantic Evaluation (SemEval 2014), 2014, no. SemEval, pp. 235-240.

[34] M. Chernyshevich, "IHS R \& D Belarus : Cross-domain Extraction of Product Features using Conditional Random Fields," in SemEval, 2014, no. SemEval, pp. 309-313.

[35] Z. Toh and J. Su, "NLANGP at SemEval-2016 Task 5: Improving Aspect Based Sentiment Analysis using Neural Network Features," Proc. SemEval-2016, vol. 2015, no. Subtask 1, pp. 282-288, 2016.

[36] N. Reimers and I. Gurevych, "Reporting Score Distributions Makes a Difference: Performance Study of LSTM-networks for Sequence Tagging," pp. 338-348, 2017. 
Figure 1 (on next page)

Overview of the MCNN architecture 
Output Layer

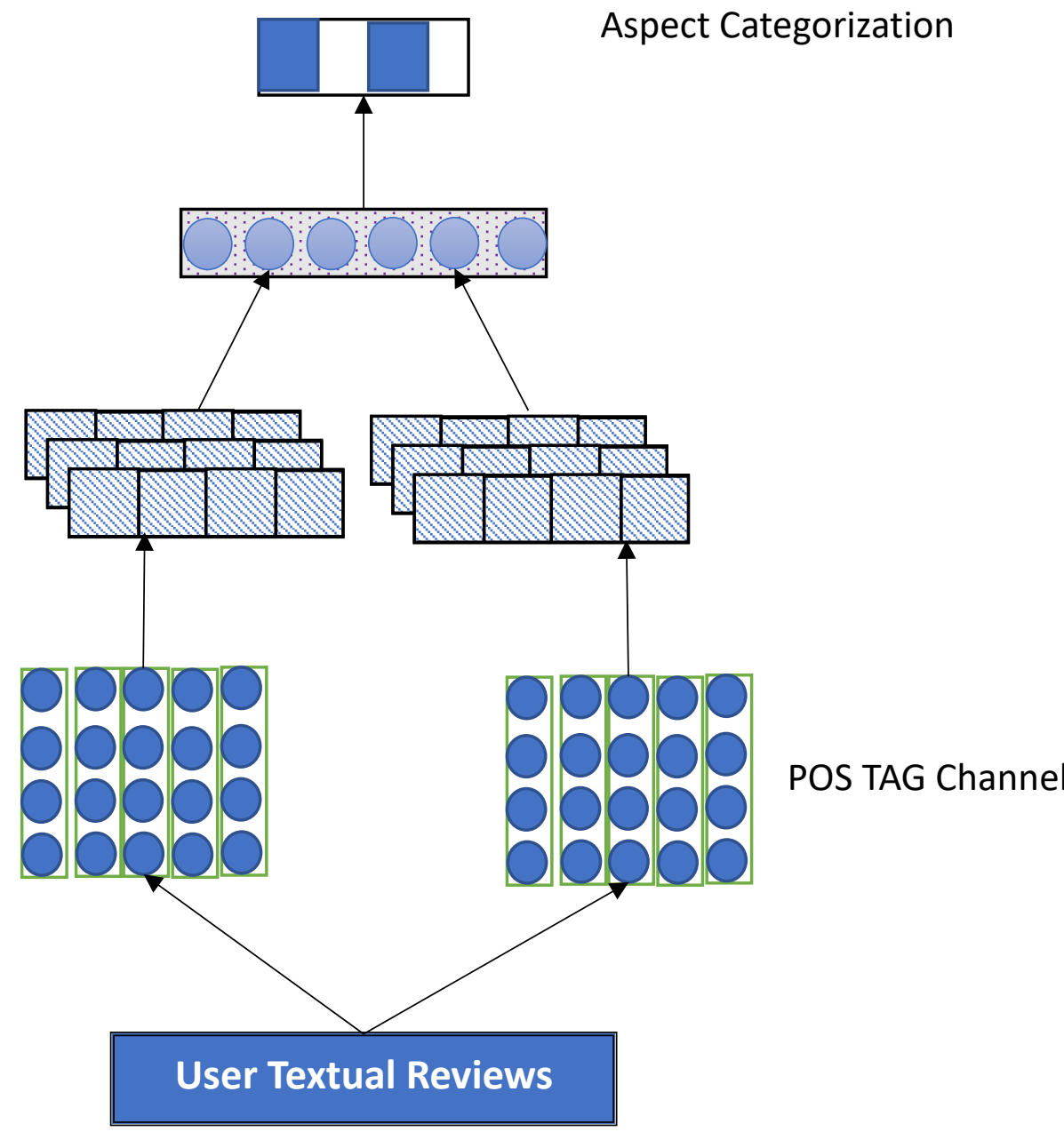


Figure 2 (on next page)

Performance of our model variants in terms of F1 score accuracy.

Each point indicates an F1 score performance computed in percentage (\%) 


\section{PeerJ Computer Science}

\section{Manuscript to be reviewed}

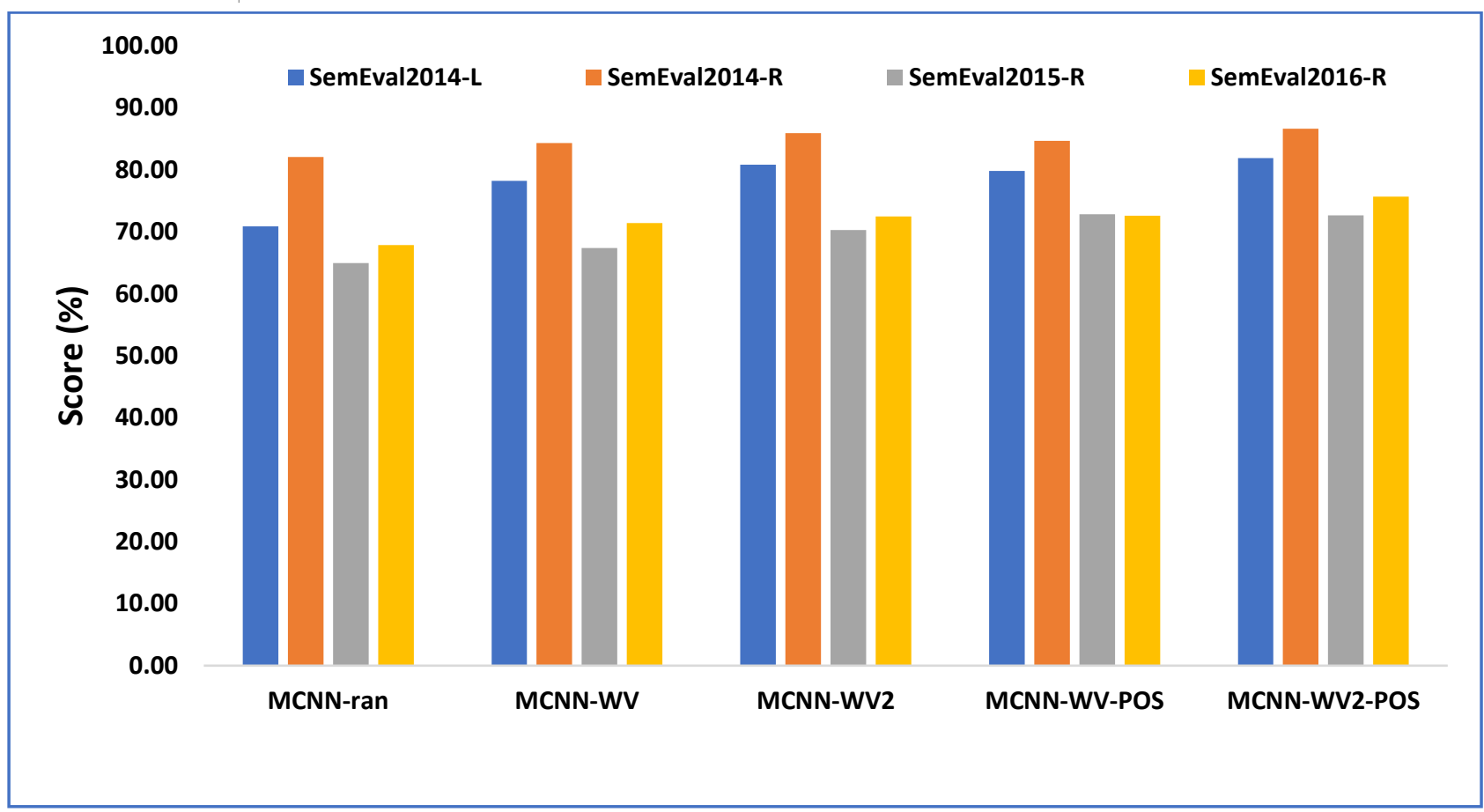


Figure 3 (on next page)

Performance of the different model variants in terms of recall and precision.

Each point shows precision and recall performance measured in percentage (\%) 


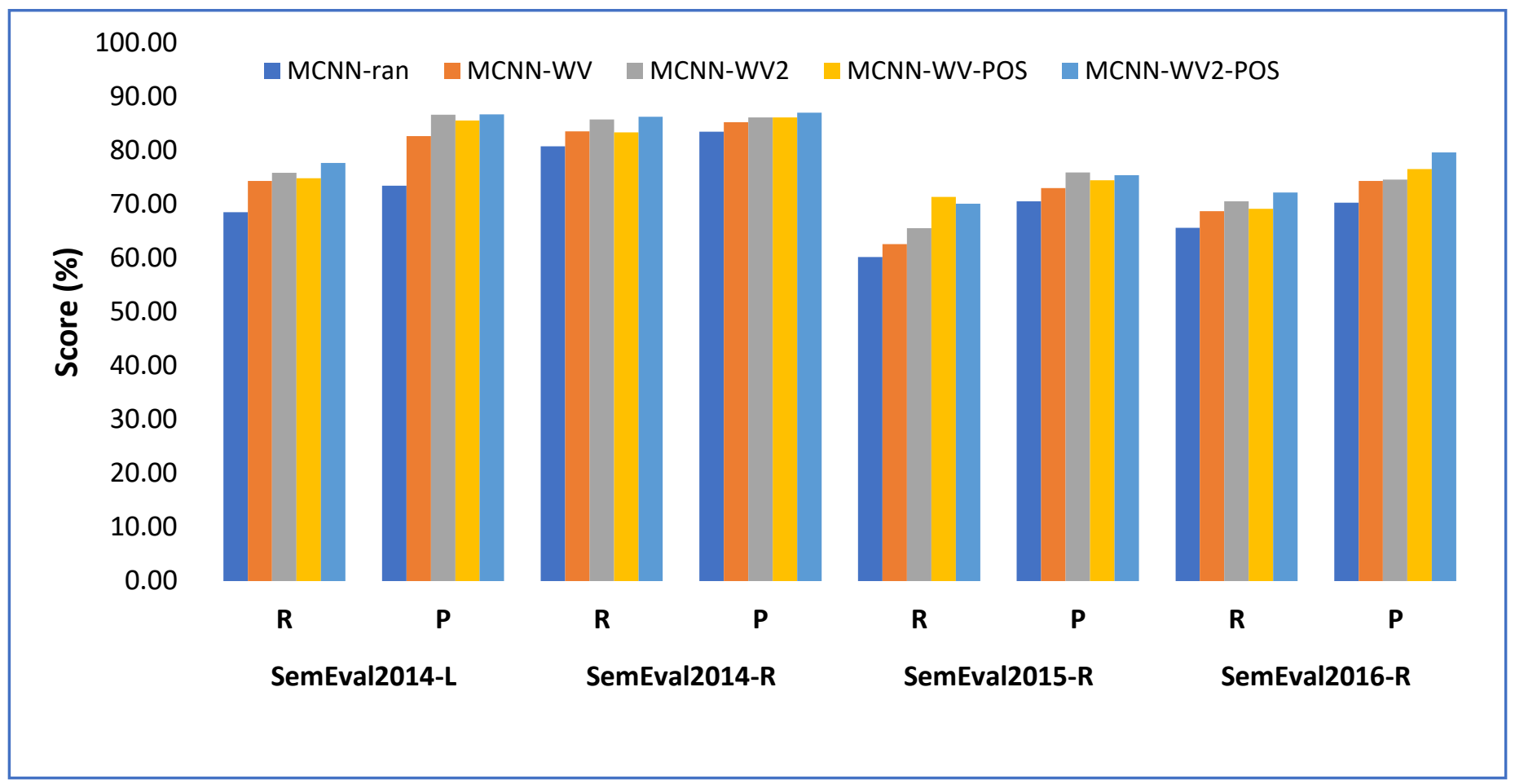


Figure 4(on next page)

F1 score of the MCNN-WV2-POS Variant of our model on different word embedding dimensions.

Each point shows the F1 performance measured in percentage (\%) 


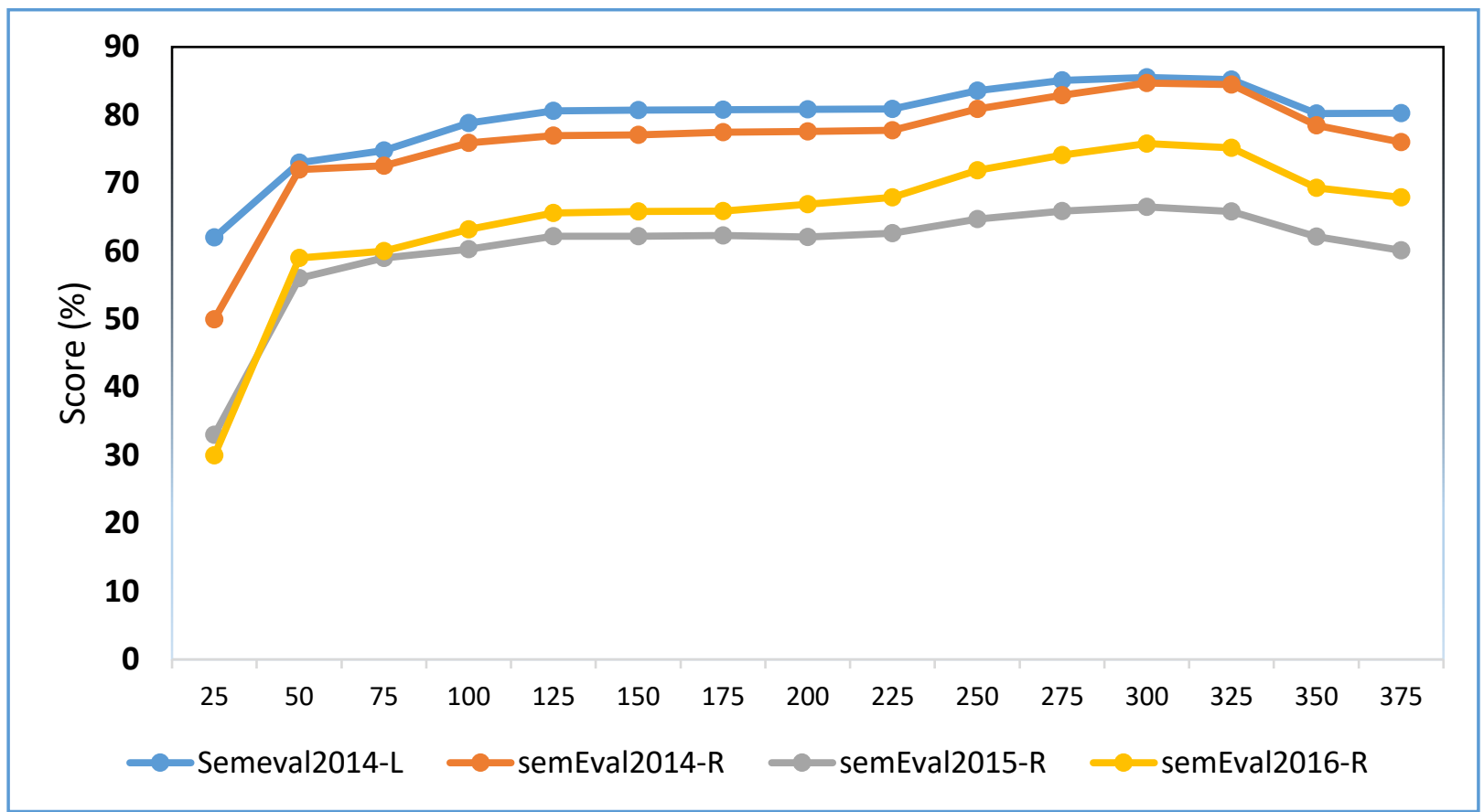




\section{Table $\mathbf{1}$ (on next page)}

SemEval challenge datasets showing the number of sentences and the aspect terms. $\mathrm{L}$ and $\mathrm{R}$ represent the laptop and restaurant domains respectively. 
1

2

3

4

\begin{tabular}{cl|l|l|l|l}
\hline \multirow{2}{*}{5} & \multicolumn{2}{|c|}{ Train } & \multicolumn{2}{c}{ Test } \\
\cline { 3 - 6 } 6 & Sentence & Aspect & Sentence & Aspect \\
\cline { 2 - 6 } & SemEval2014-L & 3041 & 2358 & 800 & 654 \\
7 & SemEval2014-R & 3045 & 3693 & 800 & 1134 \\
& SemEval2015-R & 1315 & 1192 & 685 & 678 \\
& SemEval2016-R & 2000 & 1743 & 676 & 622 \\
\hline
\end{tabular}




\section{Table 2 (on next page)}

Examples of Aspect and Aspect Terms word distribution in the laptop and restaurant domains. 
1

2

3

\begin{tabular}{l|l|l}
\hline Domain & Aspect & Aspect terms \\
\hline Laptop & Price & $\begin{array}{l}\text { price, regret, deal, Money, store, stars, gift, penny, } \\
\text { worth } \\
\text { Warranty, center, policy, support, repair, service, } \\
\text { extended, longer, contact } \\
\text { exterior, wheels, Plastic, wheel, design, interior, wheels, } \\
\text { clean, good }\end{array}$ \\
\hline Restaurant & Service & $\begin{array}{l}\text { manager, owner, Staff, workers, employees, } \\
\text { messenger, chefs, Cleaner } \\
\text { crispy, tender, Chicken, beef, shrimp, curry, tuna, egg, } \\
\text { onions } \\
\text { setting, décor, Lighting, wall } \\
\text { elegant, cool, nice, trendy }\end{array}$ \\
\hline
\end{tabular}

4 


\section{Table 3 (on next page)}

Comparison results of our best performing model variants in terms of F1 scores (\%) with the state-of-the-art methods 


\begin{tabular}{l|cccc}
\hline Model & SemEval2014-L & SemEval2014-R & SemEval2015-R & SemEval2016-R \\
\hline HIS_RD & 74.55 & 79.62 & - & - \\
NLANGP & - & - & 67.12 & 72.34 \\
DLIREC & 73.78 & 84.01 & - & - \\
WDEmb & 75.16 & 84.97 & 69.73 & - \\
RNCRF+F & 78.42 & 84.93 & - & - \\
CMLA & 77.8 & - & - & 73.44 \\
MIN & 77.58 & 85.29 & 70.73 & 74.49 \\
BidTreeCRF & 80.57 & 84.83 & 70.83 & \\
DTBCSNN & 75.66 & 83.97 & & 74.37 \\
DE-CNN & $\mathbf{8 1 . 5 9}$ & - & - & 72.62 \\
\hline MCNN+WV+POS & 79.84 & 84.69 & $\mathbf{7 2 . 8 4}$ & $\mathbf{7 5 . 7 1}$ \\
MCNN+WV2+POS & 80.63 & $\mathbf{8 6 . 8 9}$ & 72.65 & - \\
\hline
\end{tabular}

4 


\section{Table 4 (on next page)}

Comparison results of the different variants of our model in terms of recall, precision and F1 score (\%) performance. 
1

2

3

\begin{tabular}{|c|c|c|c|c|c|c|c|c|c|c|c|c|}
\hline \multirow{2}{*}{ Variant } & \multicolumn{3}{|c|}{ SemEval2014-L } & \multicolumn{3}{|c|}{ SemEval2014-R } & \multicolumn{3}{|c|}{ SemEval2015-R } & \multicolumn{3}{|c|}{ SemEval2016-R } \\
\hline & $\mathrm{R}$ & $\mathrm{P}$ & $\mathrm{F}$ & $R$ & $P$ & $\mathrm{~F}$ & $R$ & $P$ & $\mathrm{~F}$ & $R$ & $P$ & $\mathrm{~F}$ \\
\hline MCNN+ Rand & 68.50 & 73.41 & 70.87 & 80.76 & 83.45 & 82.08 & 60.20 & 70.50 & 64.94 & 65.61 & 70.25 & 67.85 \\
\hline$M C N N+W V$ & 74.30 & 82.65 & 78.25 & 83.50 & 85.20 & 84.34 & 62.60 & 73.01 & 67.41 & 68.71 & 74.32 & 71.40 \\
\hline MCNN+WV2 & 75.85 & 86.61 & 80.87 & 85.71 & 86.14 & 85.92 & 65.54 & 75.87 & 70.33 & 70.56 & 74.54 & 72.50 \\
\hline MCNN+WV+POS & 74.85 & 85.54 & 79.84 & 83.32 & 86.10 & 84.69 & 71.32 & 74.43 & 72.84 & 69.12 & 76.50 & 72.62 \\
\hline MCNN+WV2+POS & 77.65 & 86.65 & 81.90 & 86.24 & 87.01 & 86.62 & 70.08 & 75.41 & 72.65 & 72.17 & 79.61 & 75.71 \\
\hline
\end{tabular}

